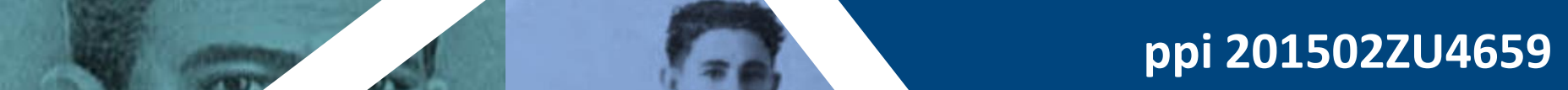

Esta publicación científica en formato digital es continuidad de la revista impresa

SSN 0254-0770/e-ISSN 2477-9377/Depósito legal pp $197802 Z U 38$

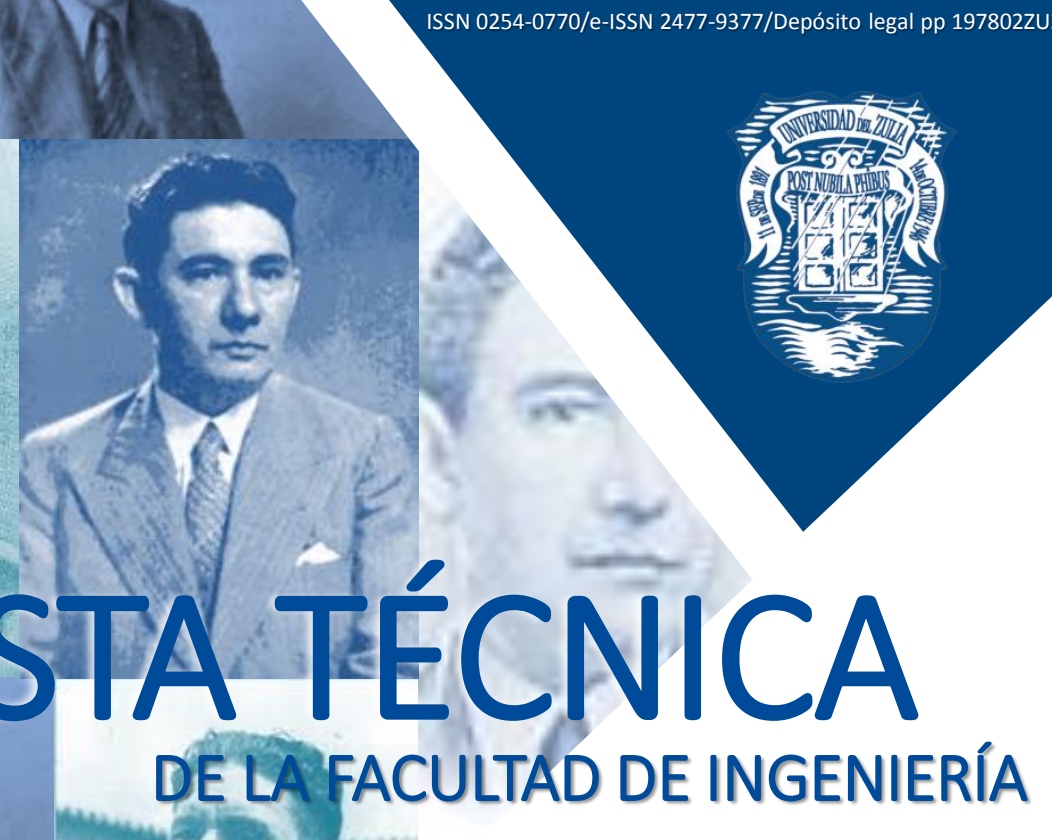
que está indizada en las publicaciones de referencia y comentarios:

- REDALYC

- REDIB

- SCIELO

- DRJI

- INDEX COPERNICUS INTERNATIONAL

- LATINDEX

- DOAJ

- REVENCYT

- CHEMICAL ABSTRACT

- MIAR

- aerospace dATABASE

- CIVIL ENGINEERING ABTRACTS

- METADEX

- COMMUNICATION ABSTRACTS

- ZENTRALBLATT MATH, ZBMATH

- ACTUALIDAD IBEROAMERICANA

- BIBLAT

- PERIODICA

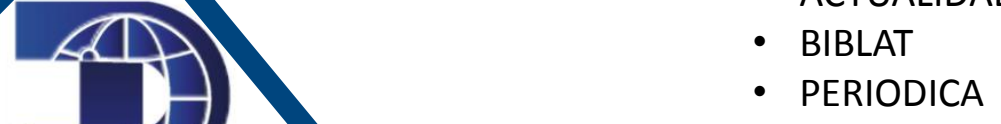




\title{
Descomposición Modular de Máquina de Estados Finita Extendida para Control Automático de un Sistema de Relevo Dual de Bombas de Pistón
}

\author{
Jorge Luis Rojas D'Onofrio \\ PDVSA-INTEVEP, Los Teques, C.P. 1201, Venezuela. \\ Autor de correspondencia: rojasj11@pdvsa.com \\ https://doi.org/10.22209/rt.v45n1a01
}

Recepción: 07 de enero 2021 | Aceptación: 23 de agosto de 2021 | Publicación: 28 de diciembre de 2021

\begin{abstract}
Resumen
Se abordó el problema de diseñar e implementar la lógica de control del sistema inyección en modo de flujo continuo para dos bombas de pistón, para un equipo de pruebas de desplazamiento de fluidos en medio poroso, con el fin de aumentar el tiempo productivo del equipo. Se planteó como objetivo el desarrollo de modelos del sistema de control comprensibles para el usuario y que a su vez facilite la depuración y la modificación del sistema evitando una explosión en la complejidad de los modelos. En una primera etapa, se elaboró un modelo monolítico de máquina de estado finita extendida en el que se toman en cuenta todos los componentes del sistema integrado por las dos bombas y las válvulas de inyección y recarga asociadas a cada una. Posteriormente, se construyó a partir del modelo monolítico un modelo modular, compuesto por una red de dos máquinas de estados finitas extendidas, cada una asociada a una de las bombas. Al implementar la red se pudo controlar automáticamente el sistema de inyección. Gracias a este enfoque se obtuvieron modelos que proporcionan flexibilidad y legibilidad al sistema; aspectos de gran relevancia en la automatización de laboratorios.
\end{abstract}

Palabras clave: automatización de laboratorios; máquina de estados finita extendida; sistemas de eventos discretos; métodos de descomposición.

\section{Modular Decomposition of a Extended Finite State Machine for Automatic Control of a Dual Piston Pump System}

\begin{abstract}
The problem of designing and implementing the control logic of an automatic injection system, in continuous flow mode for two-piston pumps, was addressed, with the aim of increasing the productive time of a test unit for fluid displacement in a porous medium. The objective was the development of control system models that are understandable for the user and, at the same time, facilitate debugging and system modification avoiding the explosion in complexity of the models. In the first stage, a monolithic extended finite-state machine model was developed, in which all the components of the system, integrated by two-piston pumps and the injection and recharge valves associated with each one, are taken into account. Subsequently, a modular model was built from the monolithic model, consisting of a network of two extended finite-state machines, each associated with one of the pumps. This network was implemented to automatically control the injection system. Thanks to this approach models were obtained that enhance flexibility and readability to the system, which are aspects of great relevance in laboratory automation.
\end{abstract}

Key words: laboratory automation; extended finite state machines; discrete event systems; decomposition methods. 


\title{
Decomposição Modular de Máquina de Estado Finito Estendida para Controle Automático de um Sistema de Alívio de Bomba de Pistão
}

\begin{abstract}
Resumo
Foi abordado o problema de projetar e implementar uma lógica de control do sistema de injeção em modo de fluxo contínuo para duas bombas de pistão, para um equipamento de teste de deslocamento de fluido em meio poroso, a fim de aumentar o tempo produtivo do equipamento. O objetivo para desenvolver modelos dos sistemas de control que sejam compreensíveis para o usuário e que por sua vez facilitem uma depuração e modificação do sistema, evitando uma explosão na complexidade dos modelos. Numa primeira fase, foi desenvolvido um modelo monolítico de uma máquina de estado finito estendida em que são respeitados todos os componentes do sistema constituído pelas duas bombas e pelas válvulas de injeção e recarga asociadas a cada uma. Posteriormente, foi construído um modelo modular partindo do modelo monolítico, composto por uma rede de duas máquinas de estado finito estendidas, cada uma associada a uma das bombas. Ao implementar uma rede, o sistema de injeção pode ser controlado automáticamente. Graças a esta abordagem, foram obtidos modelos que proporcionam flexibilidade e legibilidade ao sistema; aspectos de grande relevância na automação de laboratórios.
\end{abstract}

Palavras-chave: automatizar laboratórios; máquina de estado finito estendida; sistemas de eventos discretos; métodos de descomposição.

\section{Introducción}

La automatización de laboratorios es una actividad de creciente importancia tanto en el área de investigación y desarrollo en general, como en la producción de fármacos y la realización de pruebas clínicas (Hawker, 2007). Esta presenta similitudes con el área de automatización industrial, así como ciertas particularidades. La automatización industrial concentra sus esfuerzos en procesos a gran escala de tipo continuo (producción de energía, distribución de agua, producción de petróleo crudo), procesos de producción por lotes (derivados del petróleo, industria química) o de producción por unidades (manufacturas), en los pueden existir miles de señales y en los que los costos y riesgos asociados dificultan las modificaciones. La automatización de laboratorios, por su lado, se enfoca en procesos a pequeña escala, en los que generalmente la salida corresponde a unidades de producto (muestras, fármacos) o a datos (mediciones, análisis), en los que se tienen decenas o cientos de señales y en los que es habitual que se realicen numerosas modificaciones en un período relativamente corto de tiempo (Liscouski, 2006).

En un sondeo durante 2008, la Asociación de Automatización de Laboratorios concluyó que uno de los principales problemas en esta área es la dificultad de traducir procedimientos manuales a algoritmos automáticos, siendo las limitaciones del software de control más acuciantes que las limitaciones del hardware (Hamilton, 2009). En este sentido, se requiere una mayor flexibilidad de los sistemas de control automático, así como una mayor legibilidad y explicabilidad de la programación realizada en estos sistemas de control. La modularidad del sistema de control es una característica que se ha asociado tanto a la flexibilidad de los sistemas como a la explicabilidad de los algoritmos y programas de control (Friedrich et al., 2015).

Durante el 2016 se desarrolló un sistema automático de relevo de bombas de pistón para inyección continua (Rojas-D'Onofrio et al., 2018), como parte de los trabajos de automatización del equipo №3 del Laboratorio de Síntesis de Polímeros de PDVSA Intevep (Rojas-D’Onofrio, 2019). Se trata de un equipo para pruebas de desplazamiento de fluidos utilizado para evaluación de sistemas poliméricos en medios porosos en las condiciones de yacimiento. Está dividido en tres secciones: la sección de inyección que se describe en el presente artículo, la sección de prueba que contiene al medio poroso y en la que se realizan mediciones de presión mientras se inyectan fluidos, y una sección de salida de fluidos en la que se contabiliza el material que sale del medio poroso para hacer balances de masa. Las pruebas permiten conocer el efecto de ciertos productos químicos sobre el desplazamiento de diferentes fluidos (en este caso crudo y agua) en el yacimiento. El sistema automático de relevo se elaboró utilizando el formalismo de las máquinas de estados finitas extendidas (MEFE), las cuales fueron implementadas utilizando el entorno LabVIEW. 
Las máquinas de estados finitas (MEF) clásicas, también llamadas autómatas finitos, son de uso extendido en el desarrollo de dispositivos electrónicos y aplicaciones informáticas de diversos tipos, así como en el análisis teórico de sistemas computacionales. Además de los elementos que componen a las MEF clásicas, las MEFE incluyen el uso de variables para el registro de información adicional, así como el uso de condiciones lógicas en las transiciones de un estado a otro (Alagar y Periyasami, 2011) y se utilizan ampliamente para especificaciones de software, como por ejemplo los lenguajes SDL (Specification and Description Language), UML (Unified Modeling Language) y diferentes tipos de tablas de estado o statecharts (El-Fakih et al., 2016).

Dentro del área de automatización de sistemas de eventos discretos, los autómatas finitos han sido ampliamente utilizados para la teoría de control supervisorio o marco de Ramadge y Wonham (1989). Dentro de este marco, recientemente se han desarrollado trabajos que incorporan modelos de MEFE, así como métodos de descomposición en sistemas modulares (Mohajerani et al., 2015; Malik y Texeira, 2016; 2020). Sin embargo, esta teoría ha tenido un número limitado de aplicaciones (Wonham et al., 2017), en buena parte debido a la carencia de aplicaciones informáticas de uso comercial que proporcionen herramientas de diseño y modelado basados en esta teoría, a las dificultades de diseño y modelado que implica, y a su enfoque basado en la inhibición de eventos predefinidos y limitaciones en el tipo de especificaciones que pueden ser modeladas. Debido a esto, los aportes de la teoría de control supervisorio no se corresponden con los requerimientos abordados en el presente trabajo.

Las MEFE han sido definidas de diferentes maneras en la bibliografía (Harel, 1987; Cheng y Krishnakumar, 1993; Alagar y Periyasami, 2011; Godena y Strmcnik, 2018, Foster et al., 2020), dependiendo de las aplicaciones o del enfoque teórico utilizado. En el presente trabajo se utiliza una definición formal, que deriva de las herramientas de software utilizadas para el desarrollo del sistema de control (National Instruments, 2020).

A continuación se presenta la definición formal de MEFE utilizada, así como la definición formal de red MEFE, las cuales se emplean para describir el sistema de control desarrollado.

\section{Máquina de estados finita extendida}

Una MEFE es una lista ordenada de 6 elementos (6-tupla) $M=\left(E, e_{0}, I, O, T, S\right)$ en donde:

1. $E$ es un conjunto de estados simbólicos.

2. $e_{0}$ es el estado inicial $(E)$.

3. I es un conjunto de variables de entrada, las cuales pueden ser de diferente tipo, como por ejemplo lógicas (booleanas), numéricas (enteras, racionales, reales) o simbólicas.

4. $O$ es un conjunto de variables de salida, las cuales pueden ser de diferente tipo, como por ejemplo lógicas (booleanas), numéricas (enteras, racionales, reales) o simbólicas.

5. $T$ es un conjunto de transiciones. Cada transición $t \in T$ es una relación de tipo $\left(e^{-}, c\right) \rightarrow e^{+}$que asocia un estado previo $e^{-}$a un estado posterior $e^{+}$, a través de una condición $c$. La condición $c$ es una condición lógica construida a partir de variables de entrada $i \in I$, constantes de diferente tipo (lógicas, numéricas, simbólicas u otras), conectores lógicos, operadores aritméticos y operadores de comparación.

6. $S$ es una función de orden superior $E \rightarrow F$ que asocia a cada estado $e$ una función $f$ de las salidas de este estado. En la mayoría de los casos es conveniente representar esta función como un conjunto de pares ordenados $(e, f)$. La función $f: O \rightarrow V$ asocia a cada variable de salida $o$ un valor $v$ compatible. En la mayoría de los casos es conveniente representar esta función como un conjunto de pares ordenados $(o, v)$.

A partir de $e_{0}$, una MEFE evoluciona al evaluarse las condiciones de las transiciones que se originan en el estado actual, tomando en cuenta el valor actual de las variables de entrada. Si una condición es evaluada como verdadera, entonces la MEFE evoluciona al estado de destino de la transición correspondiente. Si ninguna condición es evaluada como verdadera, la MEFE permanece en el estado actual. En el presente caso se hace la suposición de que las MEFE se diseñan de tal manera que en un momento dado solo se cumple una o ninguna condición de las transiciones de salida de un estado, es decir se trata de una MEFE determinista. El valor de las variables de salida es determinado por la función $f=S(e)$ en donde $e$ es el estado actual.

\section{Red modular de máquinas de estados finitas extendidas}


Una red de máquinas de estados finitas extendidas (red MEFE) es una lista ordenada de 3 elementos (3tupla) $R=(M, I, O)$, en donde:

1. $M$ es un conjunto de módulos MEFE. Un módulo MEFE se define como un tipo especial de MEFE en el que el conjunto $I$ de variables entrada incluye dos variables especiales:

a. Una variable de entrada $r,(r \in I)$, que corresponde al estado de la red. El estado de la red es una lista ordenada de los estados actuales de las MEFE que conforman la red.

b. Una variable de entrada $i,(i \in I)$, que corresponde al símbolo de identificación del módulo. Esta variable se supone constante (sic) para un módulo dado de determinada red MEFE. Para dos módulos MEFE $m_{1}$ y $m_{2}$ diferentes $\left(m_{1} \neq m_{2}\right)$, las variables $i_{1}$ e $i_{2}$ correspondientes también serán diferentes $\left(i_{1} \neq i_{2}\right)$,

2. I es un conjunto de variables de entrada correspondiente a la unión de los conjuntos de variables de entrada de los módulos MEFE de la red, con la excepción de las variables especiales $r$ e $i$ de cada módulo $\operatorname{MEFE}\left(I=\left\{a: a \neq i a \neq \frac{r}{a}, i, r \in m_{3} m \in M\right\}\right)$.

3. $O$ es un conjunto de variables de salida correspondiente a la unión de los conjuntos de variables de salida de los módulos MEFE de la red ( $O=\left\{b: b \in \frac{m_{4}}{m} \in M\right\}$ ). Se hace la suposición de que para dos módulos MEFE $m_{1}$ y $m_{2}$ diferentes $\left(m_{1} \neq m_{2}\right)$, no hay variables de salida que se repitan en ambos módulos $\left(O_{1} \cap O_{2}=\emptyset\right)$.

Una red MEFE evoluciona de acuerdo a la evolución de los módulos MEFE que la conforman. Cada módulo de la red evoluciona de acuerdo al valor de las variables de entrada de la red MEFE y del estado $r$ de la red. La variable $i$ de identificación fue incluida pensando en futuros desarrollos que pudieran hacer uso de esta información para instanciar una misma MEFE. El estado $r$ de la red se actualiza cada vez que algún módulo MEFE cambia de estado. Las salidas de la red se modifican de acuerdo a los valores de salida de los módulos MEFE.

El problema abordado es el diseño e implementación de una lógica de control del sistema de inyección en modo de flujo continuo que otorgue flexibilidad y legibilidad para el usuario, evitando una posible explosión en la complejidad de los modelos. La relevancia del presente trabajo se desprende de una novedosa técnica de descomposición utilizada para generar un modelo modular del sistema de control automático desarrollado, con la cual se convirtió una MEFE monolítica, utilizada para describir el sistema de control manual previamente existente, en una red modular de dos MEFE, que facilita la comprensión, implementación, modificación y mantenimiento del nuevo sistema.

\section{Materiales y Métodos}

En esta sección se describe de manera general el proceso de inyección continua que fue automatizado, así como el diseño del sistema automático de inyección en forma de MEFE y su implementación mediante la aplicación de una técnica de descomposición de MEFE y la programación de la red MEFE resultante.

\section{Proceso de inyección de fluido mediante bombas de pistón}

El proceso incluye dos bombas de pistón encargadas de inyectar fluido en un equipo de pruebas de desplazamiento en un medio poroso (Figura 1). Cada bomba de pistón posee una cámara para almacenar el fluido, la cual debe ser cargada de manera previa a la inyección. Durante la inyección, la cantidad de fluido va disminuyendo hasta que se agota, por lo que debe recargarse, proceso durante el cual se detiene la inyección. Para continuar inyectando durante la recarga de una bomba, se utiliza una bomba de relevo, la cual ha sido previamente cargada con fluido, para que inicie la inyección apenas la primera bomba termine de inyectar. Después de efectuarse el relevo, la primera bomba inicia la recarga con el fluido almacenado en un tanque de grandes dimensiones. Una vez recargada la primera bomba, esta se mantiene en espera para relevar a la segunda bomba cuando esta última agote el fluido de su cámara de almacenamiento. Al iniciar nuevamente la primera bomba la inyección de fluido, la segunda bomba puede recargarse y el proceso puede repetirse indefinidamente. Para direccionar el fluido, cada bomba posee una válvula de inyección y una de recarga, las cuales permiten direccionar el fluido ya sea del tanque hacia la bomba o de la bomba hacia la línea de inyección (en la Figura 1 las válvulas abiertas y cerradas se muestran de color blanco y negro, respectivamente). 

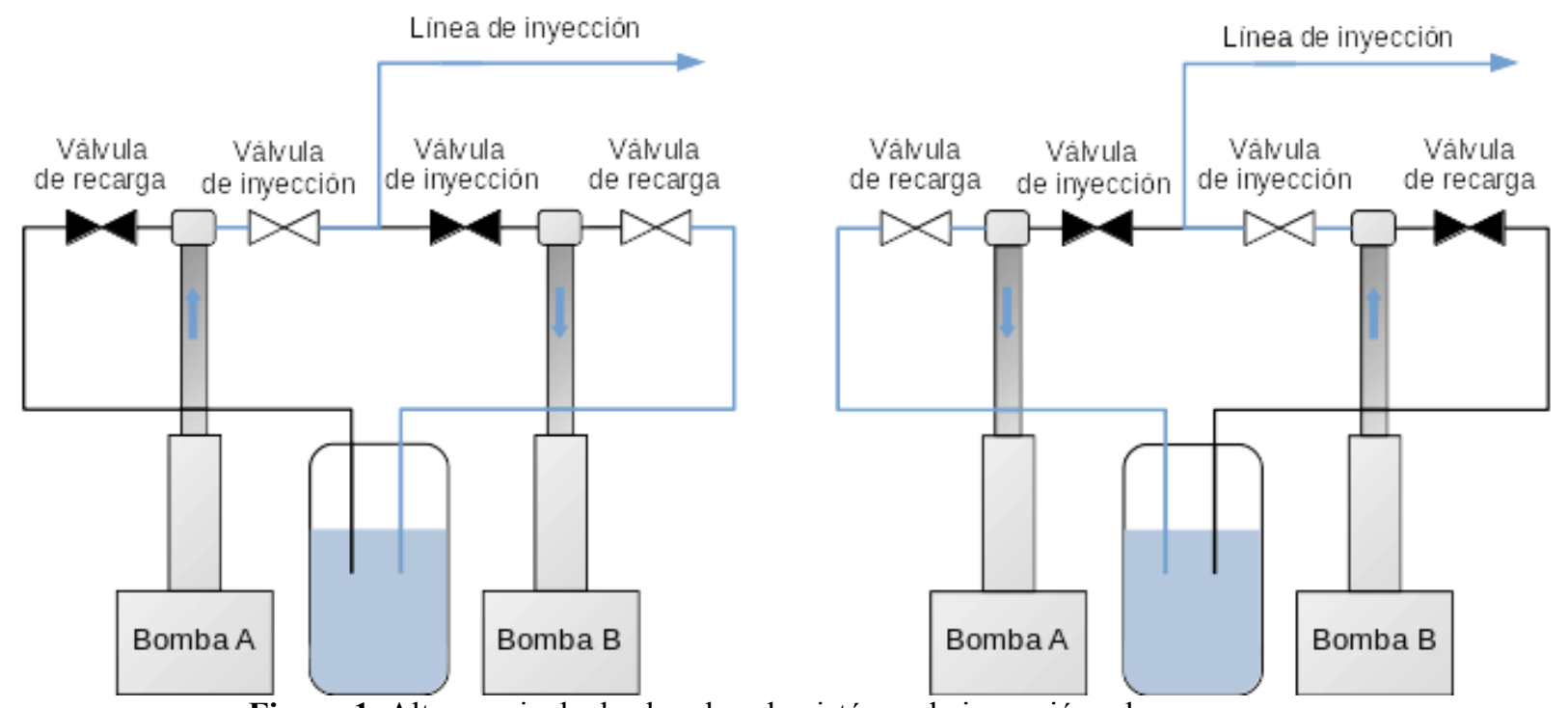

Figura 1. Alternancia de dos bombas de pistón en la inyección y la recarga.

El sistema de relevo de las bombas fue inicialmente implementado de "forma manual" por los usuarios del laboratorio, quienes se encargaban de monitorear las variables del proceso, es decir, el volumen de fluido contenido en la cámara de almacenamiento de las bombas, la apertura y cierre de las válvulas de conexión de las bombas para alinearlas con el medio poroso para la inyección o con un tanque para la recarga, y el comando para la inyección, parada o recarga de cada bomba.

Posteriormente este proceso se automatizó utilizando una computadora de escritorio (PC) conectada tanto al controlador de las bombas, para adquirir las variables necesarias y comandar las bombas, como a dispositivos para el comando de válvulas de conexión de accionamiento neumático. Se elaboró un programa para el comando automático de estos dispositivos. El programa utilizó el modelo de MEFE, el cual facilita la programación, al ser análogo a la descripción que los usuarios del laboratorio hicieron sobre su procedimiento de relevo de las bombas. En efecto, el procedimiento se caracteriza por monitorear continuamente ciertas variables durante lapsos de tiempo en los que no se realizan modificaciones a los comandos o actuadores del sistema, hasta que cierta condición provoca modificaciones súbitas que conducen a un nuevo estado del sistema. Cada lapso de tiempo durante el cual se monitorean las variables y se mantienen los valores de los comandos o señales de salida corresponde a un estado de la MEFE, mientras que las condiciones que provocan modificaciones a los valores de los comandos y señales de salida se asocian a las transiciones de la MEFE que la hacen pasar de un estado a otro.

\section{Diseño de MEFE monolítica}

Rojas D'Onofrio (2019) describe en detalle el diseño de la MEFE monolítica a partir del método manual de inyección continua utilizado por los usuarios del equipo antes de la implementación del método automático.

En la Tabla 1 se presenta la tabla de transición de estados de la MEFE monolítica. Cada estado, a excepción del denominado ModoManual o Manual, es etiquetado de acuerdo a la acción correspondiente a cada bomba. Por ejemplo, el estado Inyección/Recarga corresponde a la acción de inyección de la primera bomba (bomba A) y la acción de recarga de la segunda (bomba B). El estado ModoManual, estado inicial de la MEFE, deshabilita la inyección continua automática y permite al usuario operar las bombas.

En la Tabla 1 también se presentan las condiciones asociadas a las transiciones que permiten pasar de un estado a otro. Estas condiciones son construcciones lógicas a partir de las variables de entrada del sistema, correspondientes a los volúmenes de líquido restante en cada bomba (variables numéricas $V_{A}$ y $V_{B}$ ), al estatus de cada bomba (variables simbólicas $S_{A}$ y $S_{B}$ ), y al botón para cambiar de modo automático/manual (variable lógica o booleana $\frac{A}{M}$ ). En caso de que no se cumpla ninguna condición, se permanece en el estado actual. A cada estado se le asocian valores para las variables de salida. 
Tabla 1. Tabla de transición de estados del modelo monolítico para sistema de relevo.

\begin{tabular}{|c|c|c|c|c|c|c|}
\hline Estado & Man. & Rec./Rec. & Iny./Rec. & Iny./Esp. & Rec./Iny. & Esp./Iny. \\
\hline Manual & - & $\begin{array}{c}\mathrm{A} / \mathrm{M}=1 \wedge \\
\mathrm{V}_{\mathrm{A}}<\mathrm{V}_{\mathrm{amin}} \wedge \\
\mathrm{V}_{\mathrm{B}}<\mathrm{V}_{\mathrm{amin}}\end{array}$ & $\begin{array}{c}\mathrm{A} / \mathrm{M}=1 \wedge \\
\mathrm{V}_{\mathrm{A}} \geq \mathrm{V}_{\text {amín }} \wedge \\
\mathrm{V}_{\mathrm{A}} \geq \mathrm{V}_{\mathrm{B}}\end{array}$ & - & $\begin{array}{c}\mathrm{A} / \mathrm{M}=1 \wedge \\
\mathrm{V}_{\mathrm{B}} \geq \mathrm{V}_{\mathrm{amin}} \wedge \\
\mathrm{V}_{\mathrm{A}}<\mathrm{V}_{\mathrm{B}}\end{array}$ & - \\
\hline Rec./Rec. & $\mathrm{A} / \mathrm{M}=0$ & - & $\begin{array}{c}\mathrm{A} / \mathrm{M}=1 \wedge \\
\mathrm{S}_{\mathrm{A}}=S T O P \wedge \\
\mathrm{V}_{\mathrm{A}}=\mathrm{V}_{\text {máx }}\end{array}$ & - & $\begin{array}{c}\mathrm{A} / \mathrm{M}=1 \wedge \\
\mathrm{S}_{\mathrm{B}}=S T O P \wedge \\
\mathrm{V}_{\mathrm{B}}=\mathrm{V}_{\text {máx }} \wedge \\
\neg\left(\mathrm{S}_{\mathrm{A}}=S T O P \wedge\right. \\
\left.\mathrm{V}_{\mathrm{A}}=\mathrm{V}_{\text {máx }}\right)\end{array}$ & - \\
\hline Iny./Rec. & $\mathrm{A} / \mathrm{M}=0$ & - & $\begin{array}{c}\mathrm{A} / \mathrm{M}=1 \wedge \\
\neg\left(\mathrm{S}_{\mathrm{B}}=S T O P \wedge\right. \\
\left.\mathrm{V}_{\mathrm{B}}=\mathrm{V}_{\text {máx }}\right)\end{array}$ & $\begin{array}{c}\mathrm{A} / \mathrm{M}=1 \wedge \\
\mathrm{S}_{\mathrm{B}}=S T O P \wedge \\
\mathrm{V}_{\mathrm{B}}=\mathrm{V}_{\text {máx }}\end{array}$ & - & - \\
\hline Iny./Esp. & $\mathrm{A} / \mathrm{M}=0$ & - & - & $\begin{array}{l}\mathrm{A} / \mathrm{M}=1 \wedge \\
\mathrm{V}_{\mathrm{A}}>\mathrm{V}_{\text {mín }}\end{array}$ & $\begin{array}{l}\mathrm{A} / \mathrm{M}=1 \wedge \\
\mathrm{V}_{\mathrm{A}} \leq \mathrm{V}_{\min }\end{array}$ & - \\
\hline Rec./Iny. & $\mathrm{A} / \mathrm{M}=0$ & - & - & $\begin{array}{c}\mathrm{A} / \mathrm{M}=1 \wedge \\
\neg\left(\mathrm{S}_{\mathrm{A}}=S T O P \wedge\right. \\
\left.\mathrm{V}_{\mathrm{A}}=\mathrm{V}_{\text {máx }}\right)\end{array}$ & - & $\begin{array}{c}\mathrm{A} / \mathrm{M}=1 \wedge \\
\mathrm{S}_{\mathrm{A}}=S T O P \\
\wedge \\
\mathrm{V}_{\mathrm{A}}=\mathrm{V}_{\text {máx }}\end{array}$ \\
\hline Esp./Iny. & $\mathrm{A} / \mathrm{M}=0$ & - & $\begin{array}{l}\mathrm{A} / \mathrm{M}=1 \wedge \\
\mathrm{V}_{\mathrm{B}} \leq \mathrm{V}_{\min }\end{array}$ & - & - & $\begin{array}{c}\mathrm{A} / \mathrm{M}=1 \wedge \\
\neg\left(\mathrm{V}_{\mathrm{B}} \leq \mathrm{V}_{\min }\right)\end{array}$ \\
\hline
\end{tabular}

Man.: manual, Rec.: recarga, Iny.: inyección, Esp.: espera.

En la Tabla 2 se incluyen los valores de salida correspondientes a cada estado. Las variables $V I_{A}$ y $V I_{B}$ corresponden al estado de la válvula de inyección de cada bomba, las variables $V R_{A}$ y $V R_{B}$ al estado de la válvula de recarga de cada bomba, y las variables $C_{A}$ y $C_{B}$ al comando enviado a cada bomba correspondientes a la inyección $(R U N)$, parada $(S T O P)$ y recarga $(R E F I L L)$.

En la Figura 2 se aprecia de manera gráfica la complejidad de la MEFE. Durante la elaboración del programa se percibieron características del sistema que hacían conveniente la descomposición de la MEFE que se estaba diseñando. En particular, se constató que existía redundancia en cuanto a las acciones implementadas a través del programa y que el número de estados (6) y transiciones (14) de la MEFE era considerable. Debido a esto se procedió a diseñar un sistema modular, compuesto por dos MEFE interconectadas, a partir de la MEFE monolítica inicial.

Tabla 2. Valores de salida correspondientes a la máquina de estados del modelo monolítico.

\begin{tabular}{|c|c|c|c|c|c|c|}
\hline Estado & $\mathbf{V} \mathbf{I}_{\mathbf{A}}$ & $V I_{B}$ & $\mathbf{V R}_{\mathrm{A}}$ & $\mathbf{V R}_{\mathrm{B}}$ & $\mathbf{C}_{\mathbf{A}}$ & $\mathbf{C}_{\mathbf{B}}$ \\
\hline Modo manual & $\begin{array}{l}\text { Comando } \\
\text { anterior }\end{array}$ & $\begin{array}{l}\text { Comando } \\
\text { anterior }\end{array}$ & $\begin{array}{l}\text { Comando } \\
\text { anterior }\end{array}$ & $\begin{array}{l}\text { Comando } \\
\text { anterior }\end{array}$ & $\begin{array}{l}\text { Comando } \\
\text { anterior }\end{array}$ & $\begin{array}{l}\text { Comando } \\
\text { anterior }\end{array}$ \\
\hline $\begin{array}{l}\text { Recarga A } \\
\text { Recarga B }\end{array}$ & $\begin{array}{l}\text { Falso } \\
\text { (cerrada) }\end{array}$ & $\begin{array}{l}\text { Falso } \\
\text { (cerrada) }\end{array}$ & $\begin{array}{l}\text { Verdadero } \\
\text { (abierta) }\end{array}$ & $\begin{array}{l}\text { Verdadero } \\
\text { (abierta) }\end{array}$ & REFILL & REFILL \\
\hline $\begin{array}{l}\text { Inyección A } \\
\text { Recarga B }\end{array}$ & $\begin{array}{l}\text { Verdadero } \\
\text { (abierta) }\end{array}$ & $\begin{array}{l}\text { Falso } \\
\text { (cerrada) }\end{array}$ & $\begin{array}{l}\text { Falso } \\
\text { (cerrada) }\end{array}$ & $\begin{array}{l}\text { Verdadero } \\
\text { (abierta) }\end{array}$ & $R U N$ & REFILL \\
\hline $\begin{array}{l}\text { Inyección A } \\
\text { Espera B }\end{array}$ & $\begin{array}{l}\text { Verdadero } \\
\text { (abierta) }\end{array}$ & $\begin{array}{l}\text { Verdadero } \\
\text { (abierta) }\end{array}$ & $\begin{array}{l}\text { Falso } \\
\text { (cerrada) }\end{array}$ & $\begin{array}{l}\text { Falso } \\
\text { (cerrada) }\end{array}$ & $R U N$ & STOP \\
\hline $\begin{array}{l}\text { Recarga A } \\
\text { Inyección B }\end{array}$ & $\begin{array}{l}\text { Falso } \\
\text { (cerrada) }\end{array}$ & $\begin{array}{l}\text { Verdadero } \\
\text { (abierta) }\end{array}$ & $\begin{array}{l}\text { Verdadero } \\
\text { (abierta) }\end{array}$ & $\begin{array}{l}\text { Falso } \\
\text { (cerrada) }\end{array}$ & REFILL & $R U N$ \\
\hline $\begin{array}{l}\text { Espera A } \\
\text { Inyección B }\end{array}$ & $\begin{array}{l}\text { Verdadero } \\
\text { (abierta) }\end{array}$ & $\begin{array}{l}\text { Verdadero } \\
\text { (abierta) }\end{array}$ & $\begin{array}{l}\text { Falso } \\
\text { (cerrada) }\end{array}$ & $\begin{array}{l}\text { Falso } \\
\text { (cerrada) }\end{array}$ & STOP & $R U N$ \\
\hline
\end{tabular}

VI: válvula de inyección, VR: válvula de recarga, C: comando de la bomba. 


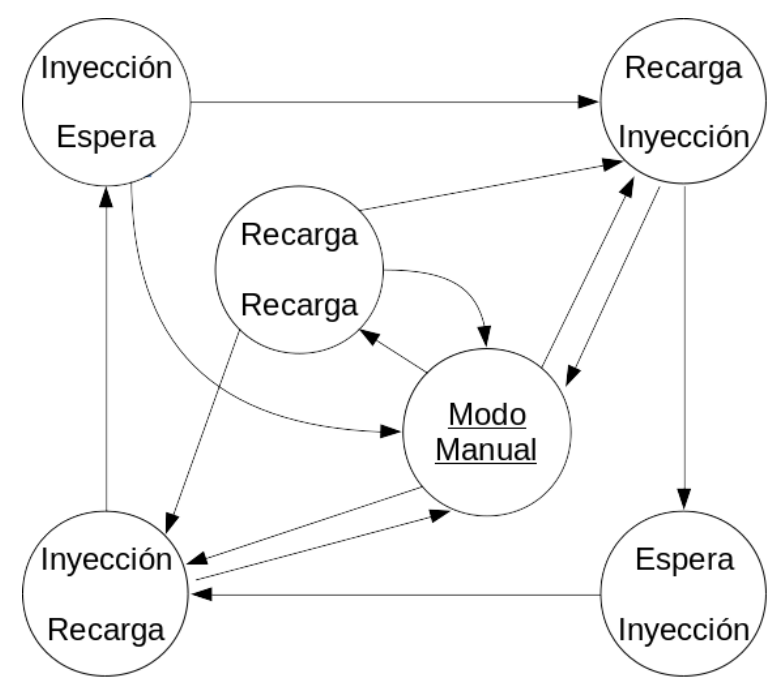

Figura 2. Máquina de estados finita extendida monolítica para inyección en modo de flujo continuo.

\section{Descomposición de MEFE}

Se tomó la decisión de diseñar, a partir de la MEFE monolítica del sistema, una MEFE (módulo) para cada bomba. Cada estado de la MEFE monolítica fue diseñado como una combinación de dos estados, cada uno de los cuales correspondería a un módulo MEFE, por lo que se generaron dos MEFE. De acuerdo a esto, para cada módulo MEFE se incluyeron los estados Manual, Inyeccion, Recarga y Espera. Cada módulo MEFE utiliza las mismas entradas de la MEFE monolítica además del estado de la otra bomba. En cambio, las señales de salidas estarían constituidas únicamente por aquellas asociadas a la bomba correspondiente.

Para generar las transiciones de cada módulo MEFE, se recorrió la tabla de transición de estados de la MEFE monolítica, determinando para cuáles transiciones se producía un cambio de estado del módulo MEFE correspondiente y para cuáles no. En el primer caso, se agrega una transición en el módulo MEFE desde el estado de origen hasta el estado de destino correspondiente. Si $c$ es la condición de la transición en la MEFE monolítica, en el módulo MEFE la condición de la transición correspondiente será $c \wedge E$, siendo $E$ el estado de origen correspondiente al otro módulo MEFE. En el segundo caso no se agrega ninguna transición al módulo MEFE. Por ejemplo, si se considera la transición de la MEFE monolítica que tiene por origen el estado Recarga/Recarga y como destino el estado Recarga/Inyección, en el caso del módulo MEFE de la bomba A no se incluirá ninguna nueva transición, ya que no existe cambio de estado (de Recarga a Recarga). En el caso de que del módulo MEFE de la bomba B, se incluye una nueva transición, con Recarga como estado de origen e Inyeccion como estado de destino. Si se denomina $c$ a la condición de la referida transición en la MEFE monolítica, la nueva transición en el módulo MEFE de la bomba $\mathrm{B}$ tendrá como condición $c\left(E_{A}=\operatorname{Recarga}\right)$, al ser ese el estado correspondiente al módulo MEFE de la bomba A en el estado de origen de la transición de la MEFE monolítica.

En la Tabla 3 se muestra la tabla de transición de estados del módulo MEFE de la bomba A, construida mediante el procedimiento descrito con algunas simplificaciones. Debido a que no existe ambigüedad, las transiciones que tienen como origen o como destino el estado ModoManual, no incluyen en su condición el estado asociado al otro módulo MEFE (bomba B). En la implementación de los módulos MEFE, se incluyeron algunas simplificaciones adicionales.

El módulo MEFE correspondiente a la bomba B es similar y puede construirse intercambiando las referencias a la bomba A por las referencias a la bomba B y tomando en cuenta la prioridad que se le da a la bomba A durante la recarga inicial (Tabla 4). 
Tabla 3. Tabla de transición de estados del modelo modular para la bomba A.

\begin{tabular}{|c|c|c|c|c|}
\hline Estado & Manual & Inyección & Recarga & Espera \\
\hline Manual & - & $\begin{array}{c}\mathrm{A} / \mathrm{M}=1 \wedge \\
\mathrm{V}_{\mathrm{A}} \geq \mathrm{V}_{\text {mín }} \wedge \\
\mathrm{V}_{\mathrm{A}} \geq \mathrm{V}_{\mathrm{B}}\end{array}$ & $\begin{array}{c}\mathrm{A} / \mathrm{M}=1 \wedge \\
\neg\left(\mathrm{V}_{\mathrm{A}} \geq \mathrm{V}_{\min } \wedge\right. \\
\left.\mathrm{V}_{\mathrm{A}} \geq \mathrm{V}_{\mathrm{B}}\right)\end{array}$ & - \\
\hline Inyección & $\mathrm{A} / \mathrm{M}=0$ & - & $\begin{array}{c}\mathrm{A} / \mathrm{M}=1 \wedge \\
\mathrm{V}_{\mathrm{A}} \leq \mathrm{V}_{\min } \wedge \\
\mathrm{E}_{\mathrm{B}}=\text { Espera }\end{array}$ & - \\
\hline Recarga & $\mathrm{A} / \mathrm{M}=0$ & $\begin{array}{c}\mathrm{A} / \mathrm{M}=1 \wedge \\
\mathrm{S}_{\mathrm{A}}=S T O P \wedge \\
\mathrm{V}_{\mathrm{A}}=\mathrm{V}_{\text {máx }} \wedge \\
\mathrm{E}_{\mathrm{B}}=\text { Recarga }\end{array}$ & - & $\begin{array}{c}\mathrm{A} / \mathrm{M}=1 \wedge \\
\mathrm{S}_{\mathrm{A}}=S T O P \wedge \\
\mathrm{V}_{\mathrm{A}}=\mathrm{V}_{\text {máx }} \wedge \\
\mathrm{E}_{\mathrm{B}}=\text { Inyección }\end{array}$ \\
\hline Espera & $\mathrm{A} / \mathrm{M}=0$ & $\begin{array}{c}\mathrm{A} / \mathrm{M}=1 \wedge \\
\mathrm{V}_{\mathrm{B}} \leq \mathrm{V}_{\text {mín }} \wedge \\
\mathrm{E}_{\mathrm{B}}=\text { Inyección }\end{array}$ & - & - \\
\hline
\end{tabular}

Tabla 4. Tabla de transición de estados del modelo modular para la bomba B.

\begin{tabular}{|c|c|c|c|c|}
\hline Estado & Manual & Inyección & Recarga & Espera \\
\hline Manual & - & $\begin{array}{c}\mathrm{A} / \mathrm{M}=1 \wedge \\
\mathrm{V}_{\mathrm{B}} \geq \mathrm{V}_{\min } \wedge \\
\mathrm{V}_{\mathrm{B}}<\mathrm{V}_{\mathrm{A}}\end{array}$ & $\begin{array}{c}\mathrm{A} / \mathrm{M}=1 \wedge \\
\neg\left(\mathrm{V}_{\mathrm{B}} \geq \mathrm{V}_{\min } \wedge\right. \\
\left.\mathrm{V}_{\mathrm{B}}<\mathrm{V}_{\mathrm{A}}\right)\end{array}$ & - \\
\hline Inyección & $\mathrm{A} / \mathrm{M}$ & - & $\begin{array}{c}\mathrm{A} / \mathrm{M}=1 \wedge \\
\mathrm{V}_{\mathrm{B}} \leq \mathrm{V}_{\min } \wedge \\
\mathrm{E}_{\mathrm{A}}=\text { Espera }\end{array}$ & - \\
\hline Recarga & $\mathrm{A} / \mathrm{M}$ & $\begin{array}{c}\mathrm{A} / \mathrm{M}=1 \wedge \\
\mathrm{S}_{\mathrm{B}}=S T O P \wedge \\
\mathrm{V}_{\mathrm{B}}=\mathrm{V}_{\text {máx }} \wedge \\
\mathrm{V}_{\mathrm{A}} \neq \mathrm{V}_{\text {máx }} \wedge \\
\mathrm{E}_{\mathrm{A}}=\text { Recarga }\end{array}$ & - & $\begin{array}{c}\mathrm{A} / \mathrm{M}=1 \wedge \\
\mathrm{S}_{\mathrm{B}}=S T O P \wedge \\
\mathrm{V}_{\mathrm{B}}=\mathrm{V}_{\text {máx }} \wedge \\
\mathrm{E}_{\mathrm{A}}=\text { Inyección }\end{array}$ \\
\hline Espera & $\mathrm{A} / \mathrm{M}$ & $\begin{array}{c}\mathrm{A} / \mathrm{M}=1 \wedge \\
\mathrm{V}_{\mathrm{A}} \leq \mathrm{V}_{\min } \wedge \\
\mathrm{E}_{\mathrm{A}}=\text { Inyección }\end{array}$ & - & - \\
\hline
\end{tabular}

En la Figura 3 se muestra de manera gráfica el módulo MEFE correspondiente a cualquiera de las dos bombas. Como puede apreciarse, se trata de una MEFE de 4 estados y 9 transiciones.

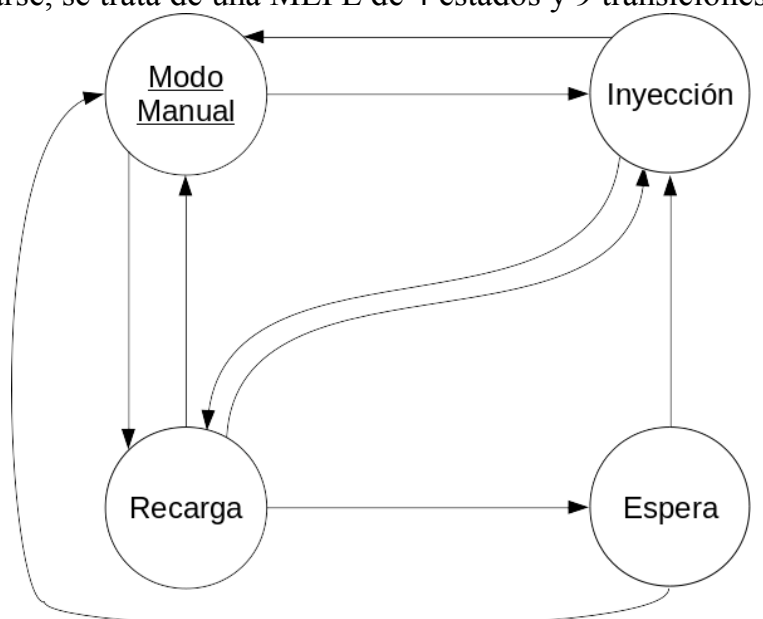

Figura 3. Máquina de estados finita extendida resultante de la descomposición modular. 


\section{Implementación de la red MEFE}

Para poner en funcionamiento al conjunto de módulos MEFE resultantes de la descomposición de la MEFE monolítica, fue necesario crear una estructura que permita la interacción entre los módulos MEFE, así como la recepción de señales de entrada y envío de señales de salida. Esta estructura corresponde a una red interconectada, en la que cada módulo MEFE recibe las variables de entrada de la red, así como información del estado del otro módulo MEFE para generar señales de salida que, agrupadas a las señales de salida del otro módulo MEFE conforman el conjunto de señales de salida de la red.

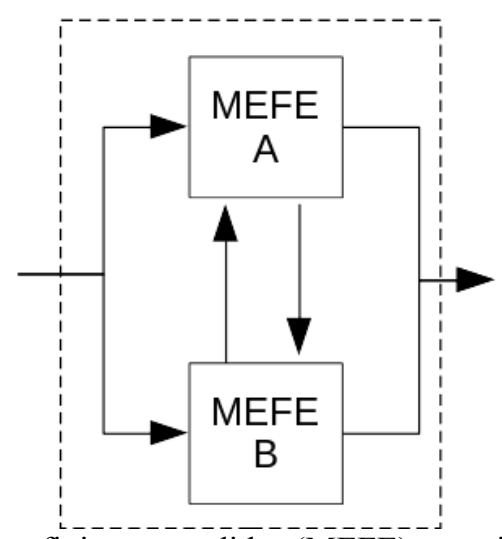

Figura 4. Red de máquinas de estados finitas extendidas (MEF), para inyección en modo de flujo continuo.

En la Figura 4 se muestra la arquitectura de la red MEFE modular. Tanto la red modular como cada uno de los módulos MEFE pueden ser implementados tanto a través de circuitos electrónicos (hardware) como mediante programas informáticos (software) utilizando diferentes tipos de lenguajes de programación. Debido a que los módulos MEFE generados son análogos, la implementación realizada en software utilizó la misma subrutina para la MEFE de cada módulo, las cuales se diferencian por las variables de entrada utilizadas.

\section{Resultados y Discusión}

El modelo de red MEFE modular fue implementado utilizando el entorno LabVIEW y probado con éxito durante el período 2016-2017, a lo largo de diez meses, con pruebas que han tenido una duración de varios días continuos, por lo que puede considerarse un sistema validado. La inyección continua puede ser verificada gracias a las mediciones de volumen de líquido inyectado y de valores de presión durante las pruebas. En las Figuras 5 y 6 se puede apreciar el comportamiento dinámico de ambas bombas para dos condiciones iniciales diferentes: un arranque con ambas bombas vacías y un arranque con las bombas con un nivel intermedio de líquido en los cilindros. Se observa tanto el valor de volumen de líquido contenido en cada bomba (VA y VB) como el flujo (FA y FB). En ambos casos las bombas manejan un máximo de $500 \mathrm{ml}$ de líquido y fueron configuradas con un flujo de inyección de $10 \mathrm{ml} / \mathrm{min}$ y un flujo de recarga de $50 \mathrm{ml} / \mathrm{min}$. El volumen mínimo $\left(\mathrm{V}_{\text {mín }}\right)$ configurado en las MEFE fue de $20 \mathrm{ml}$. En las figuras se puede, a cada instante, determinar el estado de cada MEFE si se considera que el estado Recarga corresponde a un flujo negativo de $-50 \mathrm{ml} / \mathrm{min}$, el estado Inyección a $10 \mathrm{ml} / \mathrm{min}$ y el estado Espera a $0 \mathrm{ml} / \mathrm{min}$. En el estado Manual el usuario puede asignar cualquier valor de flujo, sin embargo en los casos presentados solo se ve reflejado al inicio con un valor de $0 \mathrm{ml} / \mathrm{min}$.

En el primer caso (Figura 5) ambas bombas pasan del estado Manual al estado Recarga en el minuto 5. Ambas culminan la recarga aproximadamente en el minuto 15 y la bomba A inicia la inyección mientras que la bomba B se mantiene a la espera. Una vez alcanzado el volumen mínimo por parte de la bomba A, aproximadamente en el minuto 63, se produce el relevo por lo que la bomba B inicia la inyección mientras la bomba A inicia la recarga. En el minuto 73 aproximadamente, la bomba A culmina la recarga y se mantiene a la espera hasta el siguiente relevo en el minuto 111. Se puede apreciar en los valores de flujo que, desde el inicio de la inyección en el minuto 15, en todo momento una de las dos bombas se encuentra inyectando fluido a 10 $\mathrm{ml} / \mathrm{min}$.

En el segundo caso (Figura 6), las bombas contenían un volumen de líquido no nulo previo a la conmutación hacia el modo automático. En el minuto 5 la bomba B pasa del estado Manual al estado Inyección mientras que la bomba A pasa al estado de Recarga. Aproximadamente en el minuto 12 la bomba $\mathrm{A}$ culmina la recarga y pasa al estado Espera, mientras la bomba A continua inyectando. Luego se sucede una serie de relevos similares a los presentados en la Figura 5. De igual forma se aprecia que una vez iniciada la 
inyección, en todo momento, una de las dos bombas se encuentra inyectando, garantizando una inyección continua.

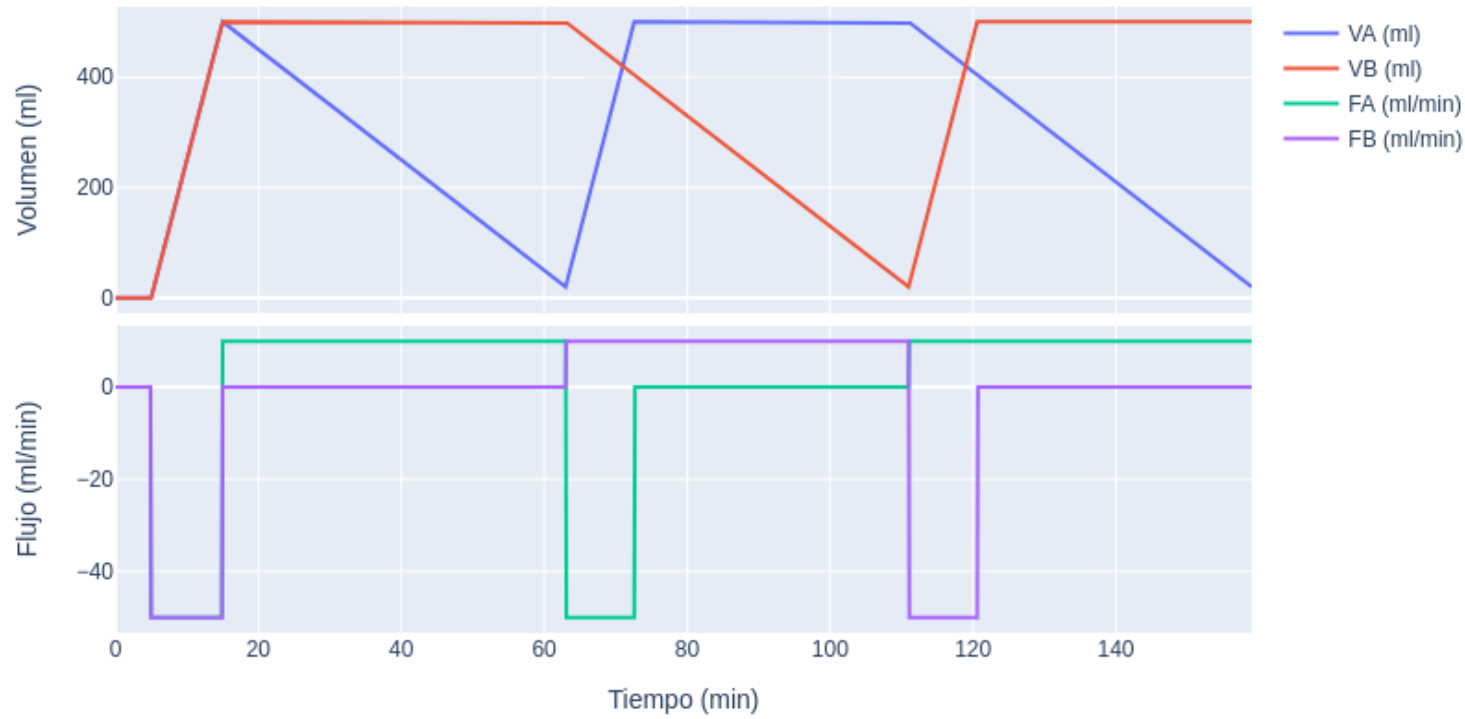

Figura 5. Comportamiento dinámico del sistema con bombas vacías al arranque (A y B).

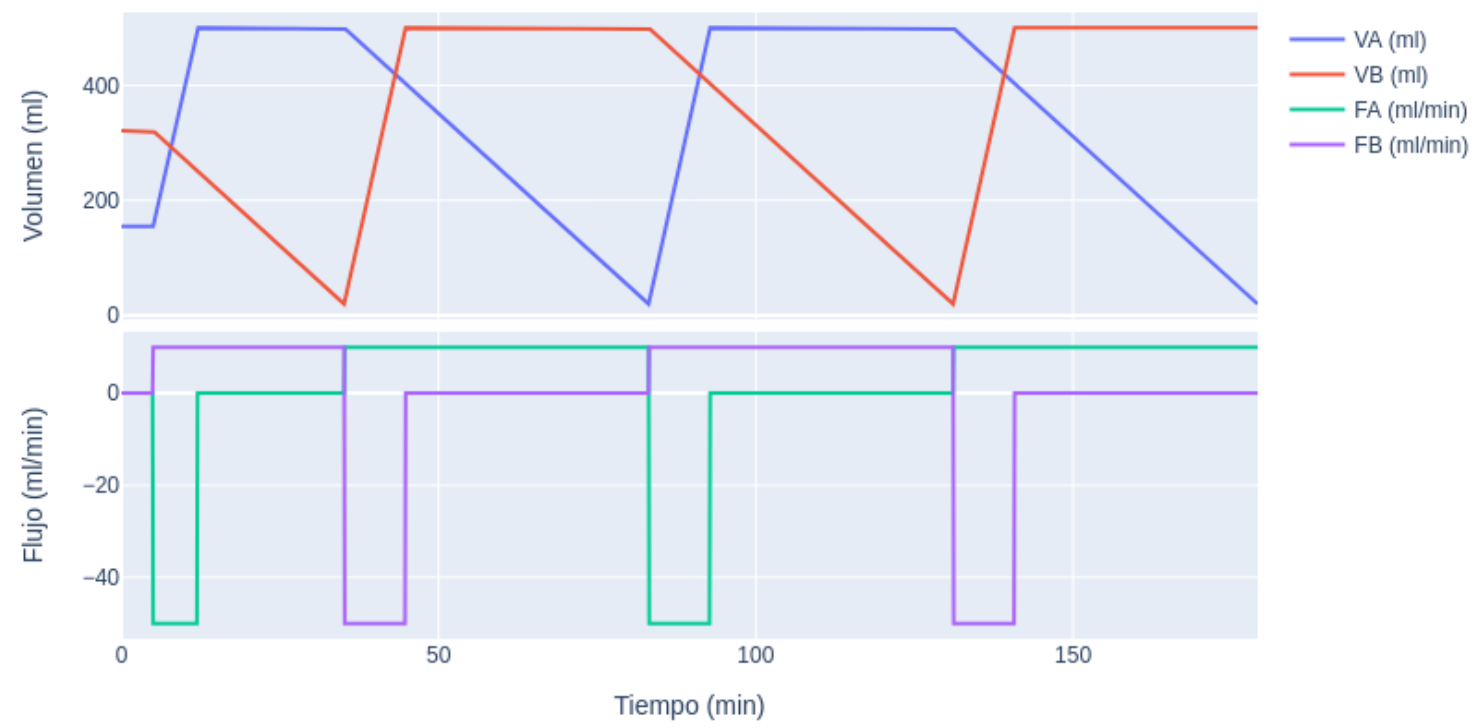

Figura 6. Comportamiento dinámico del sistema con bombas con nivel intermedio de fluido al arranque (A y B).

El desarrollo del sistema de control sirvió de base para el desarrollo de nuevas funcionalidades mediante la incorporación de estados a los módulos MEFE de la red para incorporar la funcionalidad de purga automática de aire en las bombas (Payaro-Robles, 2017). Este desarrollo aumentó el número de estados de cada módulo MEFE de 4 a 8, lo cual equivale a una MEFE monolítica de 48 estados. Esto evidencia la utilidad de trabajar con modelos modulares para evitar el problema de "explosión de estados" en modelos basados en máquinas de estados.

\section{Conclusiones}

Se desarrolló un modelo basado en MEFE para el sistema de control automático del proceso de inyección continua de fluido compuesto por dos bombas de pistón para un laboratorio de investigación y desarrollo de la industria petrolera. El desarrollo utilizó una técnica de descomposición que facilita el diseño a partir de las especificaciones y requerimientos del usuario, así como su implementación de manera modular. 
Además de cumplir con los requerimientos funcionales del proyecto, la descomposición proporcionó cualidades de flexibilidad y explicabilidad de los algoritmos, de gran relevancia para la automatización de laboratorios. El diseño del modelo monolítico permite una explicación clara de los requerimientos del usuario y está basado en la operación manual utilizada por los usuarios del equipo, quienes incluso participaron en este diseño y en su validación. El modelo modular construido facilita la implementación, depuración y modificación del sistema, especialmente en aquellos aspectos que sólo afectan a uno de los módulos. Prueba de ello es el referido desarrollo de método de purga automática de aire de las bombas, en el que fue posible expandir el número de estados de cada módulo para cumplir con nuevos requerimientos sin afectar los requerimientos iniciales y sin hacer crecer la complejidad del modelo de manera exponencial.

La metodología de descomposición de un modelo monolítico de MEFE en un modelo modular compuesto por varias MEFE que se comunican entre sí, podría ser generalizada y sistematizada, sirviendo de base para el desarrollo de algoritmos para la descomposición automática de MEFE. Este tipo de descomposición permitiría solventar el problema de explosión de estados, estudiado en la teoría de autómatas y en la teoría de control supervisorio, en casos en los que se requiera modificar un subsistema manteniendo el funcionamiento de base del sistema.

\section{Agradecimientos}

Este trabajo fue realizado en el marco de las actividades de automatización del Laboratorio de Síntesis de Polímeros, de la Gerencia de Productividad de Pozos de PDVSA INTEVEP, en particular con la participación y apoyo del Dr. Óscar Vernáez, Ing. Fabio Tamburini, Ing. Jofrank Perdomo y TSU Lorenzo Payaro.

\section{Referencias Bibliográficas}

Alagar, V., Periyasami, K. (2011). Specification of software systems, $2^{\text {nd }}$ ed. London: Springer.

Cheng, K. T., Krishnakumar, A. S. (1993). Automatic functional test generation using the extended finite state machine model. 30th international design automation conference. Dallas: ACM/IEEE, 86-91.

El-Fakih, K., Simao, A., Jadoon, N., Maldonado, J. C. (2016). An assessment of extended finite state machine test selection criteria. The Journal of Systems \& Software, 123, 106-118.

Foster, M., Brucker, A. D., Taylor, R. G., Derrick, J. (2020). A formal model of extended finite state machines. Archive of formal proofs [en línea] disponible https://ore.exeter.ac.uk/repository/bitstream/handle/10871/122936/foster.ea-efsm2020.pdf? sequence $=1 \&$ isAllowed $=y$ (consultado: 21 septiembre 2021).

Friedrich, J., Scheifele, S., Verl, A., Lechler, A. (2015). Flexible and modular control and manufacturing system. Procedia CIRP, 33, 115-120.

Godena, G., Strmcnik, S. (2018). A new state machine behavior model for procedural control entities in industrial process control systems. Journal of Information Technology and Control, 47(3), 419-430.

Hamilton, S. D. (2009). 2008 ALA survey on laboratory automation. Journal of the Association for Laboratory Automation, 14(5), 308-319.

Harel, D. (1987). Statecharts: A visual formalization for complex systems. Science of Computer Programming, $8,231-274$.

Hawker, C. D. (2007). Laboratory automation: total and subtotal. Clinics in Laboratory Medicine, 27, 749-770. Liscouski, J. G. (2006). Are you a laboratory automation engineer? Journal of the Association for Laboratory Automation, 11(3), 157-162.

Mohajerani, S., Malik, R., Fabian, M. (2015). A framework for compositional nonblocking verification of extended finite-state machines. Discrete Event Dynamic Systems, 26, 33-84.

Malik, R., Teixeira, M. (2016). Modular supervisor synthesis for extended finite-state machines subject to controllability. Proceedings of the 13th international workshop on discrete event systems. Xi'an: ACM/IEEE, 117-122. 
Malik, R., Teixeira, M. (2020). Synthesis of least restrictive controllable supervisors for extended finite-state machines with variable abstraction. Discrete Event Dynamic Systems, 30, 211-241.

National Instruments (2020). Patrones de diseño de aplicaciones: máquinas de estado [en línea] disponible en: https://www.ni.com/es-cr/support/documentation/supplemental/16/simple-state-machine-templatedocumentation.html (consultado 21 septiembre 2021).

Payaro-Robles, L. J. (2018). Desarrollo de un sistema automático de detección y purga de aire en bombas de pistón. Sartenejas: Universidad Simón Bolívar.

Ramadge, P. J. G., Wonham, W. M. (1989). The control of discrete event systems. Proceedings of the IEEE, 77(1), 81-98.

Rojas-D’Onofrio, J. L., Perdomo, J., Tamburini, F., Vernáez, O., Rondón, B. (2018). Sistema de supervisión, control y adquisición de datos de equipo N3 ubicado en el laboratorio de Síntesis de Polímeros. Los Teques: PDVSA-INTEVEP.

Rojas-D’Onofrio, J. L. (2019). Sistema automático de inyección en modo de flujo continuo para dos bombas de pistón. Los Teques: PDVSA-INTEVEP.

Wonham, W. M., Cai, K., Rudie, K. (2017). Supervisory control of discrete-event systems: a brief history 1980-2015. IFAC-PapersOnLine, 50(1), 1791-1797. 


\section{REVISTA TECNICA}

DE LA FACULTAD DE INGENIERIA

UNIVERSIDAD DEL ZULIA

Vol. 45. №1, Enero - Abril, 2022

Esta revista fue editada en formato digital y publicada en Diciembre 2021, por el Fondo Editorial Serbiluz, Universidad del Zulia. Maracaibo-Venezuela

www.luz.edu.ve

www.serbi.luz.edu.ve

www.produccioncientificaluz.org 